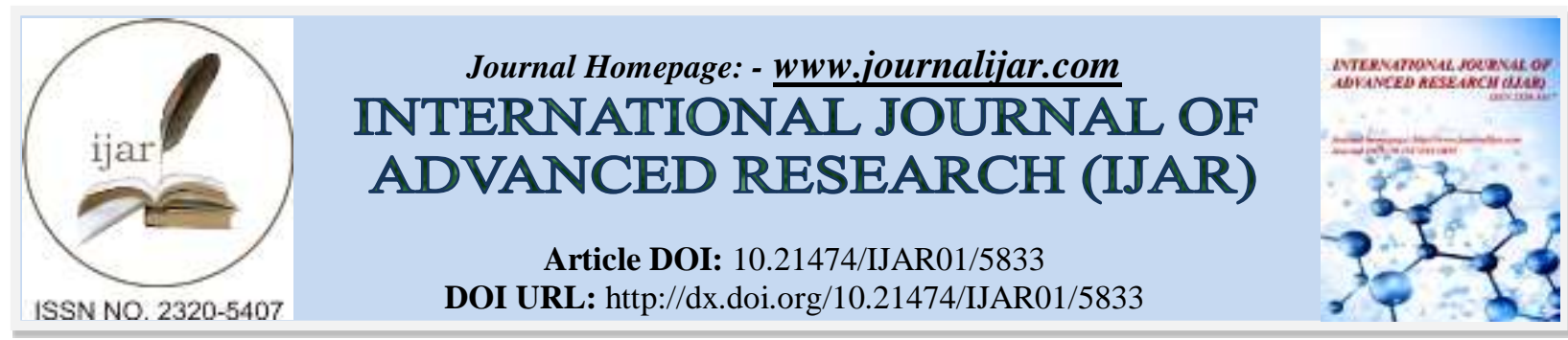

RESEARCH ARTICLE

\title{
TEST OF PERSISTENCE CHANGE UNDER THE INFLUENCE OF VARIANCE SHIFT.
}

\author{
Jiawei Sun ${ }^{1}$ and Hao Jin ${ }^{2}$. \\ 1. College of Science, Xi'an University of Science and Technology, Xi'an, China. \\ 2. Energy and Economic Research Center, Xi' an University of Science and Technology, Xi'an, China.
}

\section{Manuscript Info}

Manuscript History

Received: 11 September 2017

Final Accepted: 13 October 2017

Published: November 2017

Key words:-

Change In Persistence, Time-Varying Variance, Cusum Statistics

Found info: Supported in part by a National Natural Science Foundation of China Grant No.71473194 and No.71273206, Shaanxi Natural Science Foundation of China Grant No.2013KJXX-40 and No.2017JM1042.

\section{Abstract}

This paper studies the test of persistence change under the circumstance that the innovations have time-varying variances. We consider processes both shifting from a stationary to a unit root and the contrary change direction, the innovations of which have time-varying variances. The statistics applied in this paper is derived by S. Leybourne, R. Taylor and T. H. Kim[2007]. The limiting distributions are given under the null hypothesizes that have only variance change but persistence change and numerical simulation shows that there are severe size distortion and the power loss cannot be ignored either.

Copy Right, IJAR, 2017,. All rights reserved

\section{Introduction:-}

Since the paper of Page[1955], a vast amount of relevant articles dealing with parameter breaks have appeared in the literature and the problem of testing for and estimating of change points have attracted more and more attention among many researchers.

In recent years, a change in the persistence of a time series, which means a change in the order of integration, has come more and more into the focus of empirical and theoretical researchers. Examples of time series having change in persistence are found in De Long and Summers(1988), real output series of the U.S. and the European countries. They conjectured that these series shifted from stationarity to a unit root after WW II . They performed informal tests to find the evidence in favor of their conjecture. Beginning with Banerjee et al.(1992), several authors proposed tests for a change in persistence in the classical framework. A popular stationarity test against a break in persistence was introduced by $\mathrm{Kim}(2000)$. He has developed the residual-based ratio test against changes in persistence in a time series, focusing on the case of a shift from stochastic stationarity to difference stationarity, at some point in the sample. But Kim's test has the disadvantage to reject the null if the data generating process is constantly during the whole sample what is theoretically correct but not desirable. Leybourne et al.(2007) suggest a CUSUM-squares based test to solve this problem. Sibbertsen and Kruse(2009) generalized this test to the long memory framework by allowing for fractional degrees of integration.

Variance change, as what we understand intuitively, means the variance of a time series remains unchanged at a level until the change point, at which the variance changes to another level. In linear processes, a variance change in 
the observations implies a change in one of the errors and the converse is also true. Thus the test for a variance change can be performed based on the errors rather than the observations themselves. Furthermore, the test based on the errors outperforms the one based on observations since the latter is subject to serious power losses when the data is highly correlated. Thus, if the time series under investigation is stationary and invertible(see Brockwell and Davis(1991), for the definition), then the former is naturally preferred(cf. Park et al.(2000)). In fact, the ease of application of the CUSUM of squares test lies in the fact that the limiting distribution of the test statistic is the sup of a standard Brownian bridge. For more historical accounts of change-of-variance analysis, we refer to Gombay, Horvath and Huskova(1996), Inclan and Tiao(1994) and the references therein.

Naturally, we may think that since the CUSUM based statistics can be applied on both persistence change test and variance change test, is there any influence if the variance shift occurs in the innovations of a series which has a persistence change. In other words, we want to make it clear that whether the variance shift brings some disturbance when we test the persistence change and how the relationship is.

Our paper is organized as follows. In section 2, we give the models and assumptions that used in later theorems. Section 3 first provides a brief review of persistence change test by Kim and Leybourne et al and then gives the theoretical results under both the null hypothesizes and alternative hypothesizes. In section 4, Monte Carlo simulation methods are used to show the size distortion and power loss. Section 5 concludes. All proofs are given in the Appendix.

\section{Models and assumptions:-}

Among our paper, let

$y_{t}=\rho_{1} y_{t-1}+z_{t} \quad t \leq[\zeta T]$

$y_{t}=\rho_{2} y_{t-1}+z_{t} \quad t>[\zeta T]$

Like many other papers, $\zeta \in[0,1]$ and $[x]$ is the integer part of $x$. More specifically, in order to taking variance change into consideration, the innovation series $\left\{z_{t}\right\}$ is defined as: $z_{t}=\sigma_{t} \varepsilon_{t}$, where $\varepsilon_{t} \sim N(0,1)$ and the component, $\sigma_{t}$, is defined in the following two ways according to the different variance change types:

I. $\sigma_{t}=\sigma_{1} \cdot 1_{\{t \leq[\lambda T]\}}+\sigma_{2} \cdot 1_{\{t>[\lambda T]\}}$ for the abrupt variance change;

II. $\sigma_{t}=\sigma_{1}+\left(\sigma_{2}-\sigma_{1}\right) \cdot \min \left\{\frac{t-[\lambda T]}{T-[\lambda T]}, 0\right\}$ for the time-varying change.

In both the two types, $1\{\cdot\}$ is the indicator function and $\lambda \in[0,1]$ is the variance break point. $\sigma_{1}$ and $\sigma_{2}$ are set to be constants.

Assumption 1:- The variance term $\left\{\sigma_{\mathrm{t}}\right\}$ satisfies the relation:

$$
\sigma([\mathrm{sT}])=\omega(\mathrm{s})
$$

where $\omega(\cdot)$ is a non-stochastic function with a finite number of points of discontinuity; more over, $\omega(\cdot)>0$ and satisfied a (uniform) first-order Lipschiz condition except at the points of discontinuity.

Assumption 2:- The process $\left\{\varepsilon_{\mathrm{t}}\right\}$ satisfies the following conditions:

1. $\mathrm{E}\left(\varepsilon_{\mathrm{t}}\right)=0$

2. $E|\varepsilon|^{\gamma+\varepsilon}<\infty$ for some $\gamma>2$

3. $\left\{\varepsilon_{\mathrm{t}}\right\}$ is $\varphi-$ mixing with mixing coefficients $\varphi_{\mathrm{m}}$ satisfies $\sum_{\mathrm{m}=1}^{\infty} \varphi_{\mathrm{m}}^{1-2 / \gamma}<\infty$

4. The long-run variance $\lambda_{\varepsilon}=\sum_{j=0}^{\infty} E\left[\varepsilon_{j+1} \varepsilon_{1}\right]$ is strictly positive and finite

According to Giuseppe[2004], the Assumption 1 means that the variance function $\omega(\cdot)$ is square-integrable and bounded, i.e., $\int_{0}^{1} \omega(s)^{2} d s<\infty$; moreover, it can have up to a finite number of jumps. For instance, the function $\omega(s)=\sigma_{0}+\left(\sigma_{1}-\sigma_{0}\right) 1_{\{t>[\tau T]\}}$ corresponds to the single break model with a variance shift at time $[\tau T]$.

Assumption 2 has been used by Phillips[1987], Phillips and Perron[1988] and Phillips and Solo[1992] to derive limiting behavior of a stochastic process and allow for a broad class of weakly dependent time series. 
The null hypothesizes in our paper is as follows: $y_{t}$ is a unit root process throughout the sample period, denoted as $H_{0}$. The two corresponding alternative hypothesizes are: $y_{t}$ maintains stationarity of constant persistence until some period, after which it becomes a unit root process, denoted by $H_{01} \cdot y_{t}$ is a unit root process until [కT], after which it becomes a constant persistence series, denoted by $H_{10}$. In all the cases we explain above, the two types of variance change have always exists.

Now we write these hypothesizes below:

$$
\begin{aligned}
& H_{0}: \rho_{1}=\rho_{2}=1 \\
& H_{01}: \rho_{1}<\rho_{2}=1 \\
& H_{10}: 1=\rho_{1}<\rho_{2}
\end{aligned}
$$

\section{Theoretical results:-}

Before giving the limiting distributions under the null hypothesizes, we first briefly introduce the statistics that derived by Leybourne et al[2006] used to test change in persistence. In their paper,

$$
\begin{gathered}
K^{f}(\tau)=\frac{[\tau T]^{-2} \sum_{t=1}^{[\tau T]} \tilde{z}_{0, t}^{2}}{\hat{\sigma}_{f}^{2}(\tau)} \\
K^{b}(\tau)=\frac{(T-[\tau T])^{-2} \sum_{t=[\tau T]+1}^{T} \tilde{z}_{1, t}^{2}}{\hat{\sigma}_{b}^{2}(\tau)}
\end{gathered}
$$

The denominator of the above two statistics are estimators of the long-run variance and defined as followed:

$$
\begin{array}{ll}
\hat{\sigma}_{f}^{2}(\tau)=\hat{\gamma}_{0}+2 \sum_{s=1}^{m} \omega_{s, m} \hat{\gamma}_{s} & \hat{\gamma}_{s}=[\tau T]^{-1} \sum_{t=1}^{[\tau T]} \Delta \tilde{z}_{0, t} \Delta \tilde{z}_{0, t-s} \\
\hat{\sigma}_{b}^{2}(\tau)=\check{\gamma}_{0}+2 \sum_{s=1}^{m} \omega_{s, m} \check{\gamma}_{s} & \check{\gamma}_{s}=[\tau T]^{-1} \sum_{t=[\tau T]+1}^{T} \Delta \tilde{z}_{1, t} \Delta \tilde{z}_{1, t-s} \\
\omega_{s, m}=1-s l^{-1} &
\end{array}
$$

Due to the true break point is usually unknown, the consider the two-tailed test which rejects for large or small values of the statistic formed from the minimized CUSUMs of squared sub-sample OLS residuals obtained from the forward and backward realizations of the process; i.e.

$$
R=\frac{i n f_{\tau \in \Gamma} K^{f}(\tau)}{i n f_{\tau \in \Gamma} K^{b}(\tau)}
$$

For the test statistic above, $\Gamma$ is a compact subset of $(0,1)$.

Theorem 3.1:- Suppose that Assumption 1 and 2 are true, then under the null hypothesis $\mathrm{H}_{10}$, it is true that

$$
R \Rightarrow \frac{\tau^{-2} \int_{0}^{\tau} B_{\omega}(x)^{2} d x-\tau^{-3}\left(\int_{0}^{\tau} B_{\omega}(x) d x\right)^{2}}{(1-\tau)^{-2} \int_{\tau}^{1} B_{\omega}(x)^{2} d x-(1-\tau)^{-3}\left(\int_{\tau}^{1} B_{\omega}(x) d x\right)^{2}}
$$

Where, $B_{\omega}(s)=\bar{\sigma}^{-1} \int_{0}^{s} \omega(r) d B(r), \bar{\sigma}^{2}=\int_{0}^{1} \omega(r)^{2} d r$.

Theorem 3.2 Suppose that Assumption 1 and 2 are true, then under the hypothesizes $\mathrm{H}_{01}$ and $\mathrm{H}_{10}$, it is true that

1. Under $H_{01}, R=O_{p}(l / T)$

2. Under $H_{10}, R=O_{p}(T / l)$

\section{Simulation studies on size and power:-}

The simulation results in this section are based on sample size 100, 200 and 500 with 20,000 replications. Like what we discuss above, both the two types of null hypothesizes are considered and first we give their DGPs

First let's consider the case that $y_{t}$ under null $H_{0}$, we consider an $A R(1)$ process:

Where

$$
y_{t}=\mu_{0}+z_{t}
$$




$$
z_{t}=\rho z_{t-1}+\delta_{t} \varepsilon_{t}, \quad t=1,2, \ldots, T
$$

$\varepsilon_{t} \sim$ iid $N(0,1), \mu_{0}=0$ and $\rho=0.5, \delta_{t}$ equals to $\sigma_{1}$ before $[\lambda T]$ and $\sigma_{2}$ after [ $[\lambda T]$. The ratio of variance change takes the values from $2,3,1 / 2$ and $1 / 3$. The size simulation results of an abrupt variance and time-varying variance change are provided in Table 1 and 2 separately.

For a convenient discussion, we introduce the following definition: we say the variance change in a positive direction, which means $\sigma_{1} / \sigma_{2}<1$ and the negative direction is $\sigma_{1} / \sigma_{2}>1$.

Table 1:- Size With A = 5\% Under Unit Root Null With An Abrupt Variance Change

\begin{tabular}{|c|c|c|c|c|c|c|}
\hline & & \multicolumn{5}{|l|}{$\sigma_{1} / \sigma_{2}$} \\
\hline & $\lambda$ & 1 & 2 & 3 & $1 / 2$ & $1 / 3$ \\
\hline \multirow[t]{3}{*}{$\mathrm{T}=100$} & 0.3 & 0.048 & 0.187 & 0.414 & 0.143 & 0.213 \\
\hline & 0.5 & 0.051 & 0.222 & 0.487 & 0.166 & 0.314 \\
\hline & 0.7 & 0.049 & 0.203 & 0.452 & 0.174 & 0.319 \\
\hline \multirow[t]{3}{*}{$\mathrm{T}=200$} & 0.3 & 0.050 & 0.182 & 0.460 & 0.160 & 0.295 \\
\hline & 0.5 & 0.050 & 0.230 & 0.522 & 0.175 & 0.368 \\
\hline & 0.7 & 0.051 & 0.203 & 0.452 & 0.173 & 0.353 \\
\hline \multirow[t]{3}{*}{$\mathrm{T}=500$} & 0.3 & 0.049 & 0.196 & 0.479 & 0.158 & 0.326 \\
\hline & 0.5 & 0.049 & 0.235 & 0.567 & 0.180 & 0.374 \\
\hline & 0.7 & 0.052 & 0.230 & 0.527 & 0.169 & 0.342 \\
\hline
\end{tabular}

Table 2:- Size With $\mathrm{A}=5 \%$ Under Unit Root Null With A Time-Varying Variance Change

\begin{tabular}{|l|l|l|l|l|l|l|}
\hline & & $\sigma_{1} / \sigma_{2}$ & \multicolumn{5}{l|}{} \\
\hline \multirow{3}{*}{$\mathrm{T}=100$} & $\lambda$ & 1 & 2 & 3 & $1 / 2$ & $1 / 3$ \\
& 0.3 & 0.049 & 0.0104 & 0.0046 & 0.1216 & 0.1878 \\
\cline { 2 - 7 } & 0.5 & 0.050 & 0.0080 & 0.0042 & 0.1266 & 0.1884 \\
\cline { 2 - 7 } & 0.7 & 0.052 & 0.0114 & 0.0084 & 0.1050 & 0.1306 \\
\hline \multirow{3}{*}{$\mathrm{T}=200$} & 0.3 & 0.051 & 0.0122 & 0.0048 & 0.1382 & 0.1858 \\
\cline { 2 - 7 } & 0.5 & 0.051 & 0.0116 & 0.0048 & 0.1336 & 0.1934 \\
\cline { 2 - 7 } & 0.7 & 0.048 & 0.0126 & 0.0082 & 0.1112 & 0.1370 \\
\hline \multirow{3}{*}{$\mathrm{T}=500$} & 0.3 & 0.049 & 0.0110 & 0.0046 & 0.1288 & 0.1916 \\
\cline { 2 - 7 } & 0.5 & 0.049 & 0.0114 & 0.0050 & 0.1254 & 0.1922 \\
\cline { 2 - 7 } & 0.7 & 0.051 & 0.0178 & 0.0066 & 0.1136 & 0.1296 \\
\hline
\end{tabular}

From Table 1 we can conclude that: when there is an abrupt variance break occurs at some point among the series, severe size distortion can be observed. Besides, size value has positive correlation with variance change range. In other words, no matter the change direction of variance is positive or negative, the larger of the extent of variance shift, the more serious of size distortion. While things seem to be different when a time-varying variance change occurs. From table 2 we can easily see that in negative change direction cases, the size values are less than empirical size, which means the existence of negative time-varying variance changes will help to recognize if there is a persistence change. In the positive change cases, however, the trend of size values is just like that in abrupt cases in Table 1 and the only difference is the vary-time cases have lower extent.

Now we turn to the test power. Table 3, 4, 5 and 6 give the power of $H_{01}$ and $H_{10}$ with abrupt and time-varying variance changes, separately. In our simulation, $\rho$ is set to take value from $0.7,0.8,0.9$ and 0.95 . $\tau$ is the persistence change point position and equals to 0.5 . From Table 3, first we can easily get that the test power decreases as $\rho$ tends to 1, no matter the variance change's direction is positive or negative. This phenomenon isn't difficult to understand: a larger $\rho$ means our series is more similar to a unit root process and it is certainly hard for the test statistics to distinguish $\left\{y_{t}\right\}$ from a stationary series. Second, for all the change directions, test power will decrease as $\lambda \rightarrow 1$. Besides, when the change direction is negative, the test power has positive correlation with the change extent of variance. While for the positive change direction, things seem to be complex. When $\rho \geq 0.9$, power has the same change trend with negative cases; but when $\rho<0.9$, power has negative correlation with change extent of variance. From Table 4, the power values under a $\rho$ that is not so close to 1 are larger than those in the same position in Table 3 generally, which isn't hard to understand: the influence brought by a time-varying variance 
change is more gentle than by an abrupt variance change. A special attention should be paid that power values in positive change direction and $\rho<0.9$, still stay different with other cases.

Next we turn to Table 5 and 6. First we can conclude in Table 5 the test power will increase as the growth of sample size, especially for the positive change direction. The influence brought by the variance change extent looks less significant compared with Table 3 and 4 . For the positive change direction, power values have a significant growth as $\lambda \rightarrow 1$, while it is negligible for the negative change cases. But we should pay special attention to the fact that the power values in Table 6 when change direction is negative have an opposite change direction. That is to say, power value has negative correlation with $\lambda$ when there is an abrupt variance change under $H_{01}$.

Table 3:- Power under $H_{10}$ with a time-varying variance change

\begin{tabular}{|c|c|c|c|c|c|c|c|c|c|c|c|c|c|c|}
\hline \multirow{2}{*}{$\sigma_{1} / \sigma_{2}$} & \multirow[b]{2}{*}{$\lambda$} & \multirow{2}{*}{$\begin{array}{l}\rho \\
T\end{array}$} & \multicolumn{3}{|l|}{0.7} & \multicolumn{3}{|l|}{0.8} & \multicolumn{3}{|l|}{0.9} & \multicolumn{3}{|l|}{0.95} \\
\hline & & & 100 & 200 & 500 & 100 & 200 & 500 & 100 & 200 & 500 & 100 & 200 & 500 \\
\hline \multirow[t]{3}{*}{2} & $\begin{array}{l}0 . \\
3\end{array}$ & & $\begin{array}{l}0.59 \\
2\end{array}$ & $\begin{array}{l}0.80 \\
3\end{array}$ & $\begin{array}{l}0.96 \\
0\end{array}$ & $\begin{array}{l}0.45 \\
6\end{array}$ & $\begin{array}{l}0.69 \\
7\end{array}$ & $\begin{array}{l}0.93 \\
1\end{array}$ & $\begin{array}{l}0.13 \\
4\end{array}$ & $\begin{array}{l}0.26 \\
4\end{array}$ & $\begin{array}{l}0.67 \\
1\end{array}$ & $\begin{array}{l}0.02 \\
0\end{array}$ & $\begin{array}{l}0.12 \\
4\end{array}$ & $\begin{array}{l}0.38 \\
7\end{array}$ \\
\hline & $\begin{array}{l}0 . \\
5\end{array}$ & & $\begin{array}{l}0.36 \\
1\end{array}$ & $\begin{array}{l}0.69 \\
2\end{array}$ & $\begin{array}{l}0.96 \\
4\end{array}$ & $\begin{array}{l}0.17 \\
9\end{array}$ & $\begin{array}{l}0.43 \\
3\end{array}$ & $\begin{array}{l}0.87 \\
7\end{array}$ & $\begin{array}{l}0.03 \\
4\end{array}$ & $\begin{array}{l}0.08 \\
0\end{array}$ & $\begin{array}{l}0.34 \\
5\end{array}$ & $\begin{array}{l}0.00 \\
3\end{array}$ & $\begin{array}{l}0.01 \\
2\end{array}$ & $\begin{array}{l}0.10 \\
6\end{array}$ \\
\hline & $\begin{array}{l}0 . \\
7\end{array}$ & & $\begin{array}{l}0.07 \\
3\end{array}$ & $\begin{array}{l}0.28 \\
7\end{array}$ & $\begin{array}{l}0.80 \\
0\end{array}$ & $\begin{array}{l}0.01 \\
7\end{array}$ & $\begin{array}{l}0.09 \\
5\end{array}$ & $\begin{array}{l}0.52 \\
4\end{array}$ & $\begin{array}{l}0.00 \\
5\end{array}$ & $\begin{array}{l}0.00 \\
9\end{array}$ & $\begin{array}{l}0.07 \\
6\end{array}$ & $\begin{array}{l}0.00 \\
1\end{array}$ & $\begin{array}{l}0.00 \\
2\end{array}$ & $\begin{array}{l}0.00 \\
6\end{array}$ \\
\hline \multirow[t]{3}{*}{3} & $\begin{array}{l}0 . \\
3\end{array}$ & & $\begin{array}{l}0.62 \\
2\end{array}$ & $\begin{array}{l}0.83 \\
3\end{array}$ & $\begin{array}{l}0.96 \\
2\end{array}$ & $\begin{array}{l}0.49 \\
0\end{array}$ & $\begin{array}{l}0.71 \\
2\end{array}$ & $\begin{array}{l}0.94 \\
2\end{array}$ & $\begin{array}{l}0.15 \\
2\end{array}$ & $\begin{array}{l}0.27 \\
2\end{array}$ & $\begin{array}{l}0.65 \\
7\end{array}$ & $\begin{array}{l}0.02 \\
0\end{array}$ & $\begin{array}{l}0.13 \\
8\end{array}$ & $\begin{array}{l}0.42 \\
7\end{array}$ \\
\hline & $\begin{array}{l}0 . \\
5\end{array}$ & & $\begin{array}{l}0.39 \\
9\end{array}$ & $\begin{array}{l}0.69 \\
5\end{array}$ & $\begin{array}{l}0.97 \\
2\end{array}$ & $\begin{array}{l}0.20 \\
1\end{array}$ & $\begin{array}{l}0.48 \\
1\end{array}$ & $\begin{array}{l}0.88 \\
4\end{array}$ & $\begin{array}{l}0.03 \\
9\end{array}$ & $\begin{array}{l}0.08 \\
2\end{array}$ & $\begin{array}{l}0.31 \\
2\end{array}$ & $\begin{array}{l}0.00 \\
3\end{array}$ & $\begin{array}{l}0.01 \\
1\end{array}$ & $\begin{array}{l}0.13 \\
8\end{array}$ \\
\hline & $\begin{array}{l}0 . \\
7\end{array}$ & & $\begin{array}{l}0.07 \\
9\end{array}$ & $\begin{array}{l}0.31 \\
9\end{array}$ & $\begin{array}{l}0.81 \\
9\end{array}$ & $\begin{array}{l}0.01 \\
5\end{array}$ & $\begin{array}{l}0.10 \\
5\end{array}$ & $\begin{array}{l}0.56 \\
6\end{array}$ & $\begin{array}{l}0.00 \\
4\end{array}$ & $\begin{array}{l}0.00 \\
8\end{array}$ & $\begin{array}{l}0.05 \\
1\end{array}$ & $\begin{array}{l}0.00 \\
3\end{array}$ & $\begin{array}{l}0.00 \\
1\end{array}$ & $\begin{array}{l}0.00 \\
8\end{array}$ \\
\hline \multirow[t]{3}{*}{4} & $\begin{array}{l}0 . \\
3\end{array}$ & & $\begin{array}{l}0.61 \\
4\end{array}$ & $\begin{array}{l}0.83 \\
0\end{array}$ & $\begin{array}{l}0.96 \\
4\end{array}$ & $\begin{array}{l}0.50 \\
0\end{array}$ & $\begin{array}{l}0.72 \\
7\end{array}$ & $\begin{array}{l}0.94 \\
4\end{array}$ & $\begin{array}{l}0.15 \\
7\end{array}$ & $\begin{array}{l}0.28 \\
2\end{array}$ & $\begin{array}{l}0.63 \\
1\end{array}$ & $\begin{array}{l}0.01 \\
7\end{array}$ & $\begin{array}{l}0.14 \\
7\end{array}$ & $\begin{array}{l}0.44 \\
8\end{array}$ \\
\hline & $\begin{array}{l}0 . \\
5\end{array}$ & & $\begin{array}{l}0.41 \\
5\end{array}$ & $\begin{array}{l}0.71 \\
3\end{array}$ & $\begin{array}{l}0.97 \\
1\end{array}$ & $\begin{array}{l}0.23 \\
2\end{array}$ & $\begin{array}{l}0.51 \\
3\end{array}$ & $\begin{array}{l}0.89 \\
7\end{array}$ & $\begin{array}{l}0.04 \\
2\end{array}$ & $\begin{array}{l}0.08 \\
3\end{array}$ & $\begin{array}{l}0.31 \\
8\end{array}$ & $\begin{array}{l}0.00 \\
4\end{array}$ & $\begin{array}{l}0.01 \\
4\end{array}$ & $\begin{array}{l}0.15 \\
6\end{array}$ \\
\hline & $\begin{array}{l}0 . \\
7\end{array}$ & & $\begin{array}{l}0.07 \\
9\end{array}$ & $\begin{array}{l}0.34 \\
1\end{array}$ & $\begin{array}{l}0.82 \\
2\end{array}$ & $\begin{array}{l}0.01 \\
6\end{array}$ & $\begin{array}{l}0.11 \\
5\end{array}$ & $\begin{array}{l}0.58 \\
1\end{array}$ & $\begin{array}{l}0.00 \\
3\end{array}$ & $\begin{array}{l}0.00 \\
6\end{array}$ & $\begin{array}{l}0.03 \\
0\end{array}$ & $\begin{array}{l}0.00 \\
1\end{array}$ & $\begin{array}{l}0.00 \\
2\end{array}$ & $\begin{array}{l}0.00 \\
4\end{array}$ \\
\hline \multirow[t]{3}{*}{$1 / 2$} & $\begin{array}{l}0 . \\
3\end{array}$ & & $\begin{array}{l}0.47 \\
0\end{array}$ & $\begin{array}{l}0.68 \\
5\end{array}$ & $\begin{array}{l}0.88 \\
4\end{array}$ & $\begin{array}{l}0.34 \\
2\end{array}$ & $\begin{array}{l}0.58 \\
8\end{array}$ & $\begin{array}{l}0.88 \\
4\end{array}$ & $\begin{array}{l}0.19 \\
5\end{array}$ & $\begin{array}{l}0.37 \\
2\end{array}$ & $\begin{array}{l}0.78 \\
2\end{array}$ & $\begin{array}{l}0.05 \\
9\end{array}$ & $\begin{array}{l}0.08 \\
3\end{array}$ & $\begin{array}{l}0.26 \\
1\end{array}$ \\
\hline & $\begin{array}{l}0 . \\
5\end{array}$ & & $\begin{array}{l}0.23 \\
7\end{array}$ & $\begin{array}{l}0.51 \\
7\end{array}$ & $\begin{array}{l}0.90 \\
1\end{array}$ & $\begin{array}{l}0.11 \\
8\end{array}$ & $\begin{array}{l}0.31 \\
8\end{array}$ & $\begin{array}{l}0.80 \\
1\end{array}$ & $\begin{array}{l}0.02 \\
6\end{array}$ & $\begin{array}{l}0.11 \\
1\end{array}$ & $\begin{array}{l}0.46 \\
9\end{array}$ & $\begin{array}{l}0.00 \\
5\end{array}$ & $\begin{array}{l}0.02 \\
2\end{array}$ & $\begin{array}{l}0.06 \\
3\end{array}$ \\
\hline & $\begin{array}{l}0 . \\
7\end{array}$ & & $\begin{array}{l}0.05 \\
8\end{array}$ & $\begin{array}{l}0.20 \\
3\end{array}$ & $\begin{array}{l}0.70 \\
0\end{array}$ & $\begin{array}{l}0.02 \\
0\end{array}$ & $\begin{array}{l}0.07 \\
6\end{array}$ & $\begin{array}{l}0.41 \\
0\end{array}$ & $\begin{array}{l}0.00 \\
2\end{array}$ & $\begin{array}{l}0.00 \\
7\end{array}$ & $\begin{array}{l}0.11 \\
0\end{array}$ & $\begin{array}{l}0.00 \\
2\end{array}$ & $\begin{array}{l}0.00 \\
2\end{array}$ & $\begin{array}{l}0.01 \\
3\end{array}$ \\
\hline \multirow[t]{3}{*}{$1 / 3$} & $\begin{array}{l}0 . \\
3\end{array}$ & & $\begin{array}{l}0.39 \\
2\end{array}$ & $\begin{array}{l}0.61 \\
2\end{array}$ & $\begin{array}{l}0.79 \\
4\end{array}$ & $\begin{array}{l}0.31 \\
6\end{array}$ & $\begin{array}{l}0.54 \\
3\end{array}$ & $\begin{array}{l}0.80 \\
7\end{array}$ & $\begin{array}{l}0.22 \\
1\end{array}$ & $\begin{array}{l}0.42 \\
7\end{array}$ & $\begin{array}{l}0.79 \\
1\end{array}$ & $\begin{array}{l}0.06 \\
9\end{array}$ & $\begin{array}{l}0.09 \\
8\end{array}$ & $\begin{array}{l}0.26 \\
4\end{array}$ \\
\hline & $\begin{array}{l}0 . \\
5\end{array}$ & & $\begin{array}{l}0.21 \\
4\end{array}$ & $\begin{array}{l}0.46 \\
2\end{array}$ & $\begin{array}{l}0.81 \\
5\end{array}$ & $\begin{array}{l}0.12 \\
8\end{array}$ & $\begin{array}{l}0.30 \\
7\end{array}$ & $\begin{array}{l}0.72 \\
9\end{array}$ & $\begin{array}{l}0.02 \\
2\end{array}$ & $\begin{array}{l}0.13 \\
9\end{array}$ & $\begin{array}{l}0.51 \\
0\end{array}$ & $\begin{array}{l}0.01 \\
3\end{array}$ & $\begin{array}{l}0.02 \\
6\end{array}$ & $\begin{array}{l}0.07 \\
9\end{array}$ \\
\hline & $\begin{array}{l}0 . \\
7\end{array}$ & & $\begin{array}{l}0.04 \\
6\end{array}$ & $\begin{array}{l}0.16 \\
7\end{array}$ & $\begin{array}{l}0.63 \\
5\end{array}$ & $\begin{array}{l}0.01 \\
8\end{array}$ & $\begin{array}{l}0.05 \\
4\end{array}$ & $\begin{array}{l}0.32 \\
7\end{array}$ & $\begin{array}{l}0.00 \\
4\end{array}$ & $\begin{array}{l}0.00 \\
7\end{array}$ & $\begin{array}{l}0.11 \\
4\end{array}$ & $\begin{array}{l}0.00 \\
2\end{array}$ & $\begin{array}{l}0.00 \\
2\end{array}$ & $\begin{array}{l}0.00 \\
5\end{array}$ \\
\hline \multirow[t]{2}{*}{$1 / 4$} & $\begin{array}{l}0 . \\
3\end{array}$ & & $\begin{array}{l}0.31 \\
5\end{array}$ & $\begin{array}{l}0.49 \\
0\end{array}$ & $\begin{array}{l}0.70 \\
2\end{array}$ & $\begin{array}{l}0.29 \\
6\end{array}$ & $\begin{array}{l}0.49 \\
6\end{array}$ & $\begin{array}{l}0.74 \\
8\end{array}$ & $\begin{array}{l}0.23 \\
1\end{array}$ & $\begin{array}{l}0.44 \\
3\end{array}$ & $\begin{array}{l}0.79 \\
9\end{array}$ & $\begin{array}{l}0.07 \\
9\end{array}$ & $\begin{array}{l}0.10 \\
7\end{array}$ & $\begin{array}{l}0.27 \\
6\end{array}$ \\
\hline & $\begin{array}{l}0 . \\
5\end{array}$ & & $\begin{array}{l}0.19 \\
2\end{array}$ & $\begin{array}{l}0.39 \\
7\end{array}$ & $\begin{array}{l}0.72 \\
6\end{array}$ & $\begin{array}{l}0.12 \\
1\end{array}$ & $\begin{array}{l}0.27 \\
9\end{array}$ & $\begin{array}{l}0.66 \\
8\end{array}$ & $\begin{array}{l}0.02 \\
0\end{array}$ & $\begin{array}{l}0.15 \\
8\end{array}$ & $\begin{array}{l}0.54 \\
1\end{array}$ & $\begin{array}{l}0.01 \\
8\end{array}$ & $\begin{array}{l}0.02 \\
9\end{array}$ & $\begin{array}{l}0.07 \\
6\end{array}$ \\
\hline
\end{tabular}




\begin{tabular}{|l|l|l|l|l|l|l|l|l|l|l|l|l|l|l|l|l|l|}
\hline & 0. & & 0.03 & 0.12 & 0.53 & 0.01 & 0.03 & 0.26 & 0.00 & 0.00 & 0.11 & 0.00 & 0.00 & 0.00 \\
& 7 & & 2 & 4 & 7 & 0 & 9 & 0 & 4 & 8 & 6 & 2 & 2 & 5 \\
\hline
\end{tabular}

Table 4:- Power under $H_{10}$ with an abrupt variance change

\begin{tabular}{|c|c|c|c|c|c|c|c|c|c|c|c|c|c|c|}
\hline \multirow{2}{*}{$\sigma_{1} / \sigma_{2}$} & \multirow[b]{2}{*}{$\lambda$} & \multirow{2}{*}{$\begin{array}{l}\rho \\
T\end{array}$} & \multicolumn{3}{|l|}{0.7} & \multicolumn{3}{|l|}{0.8} & \multicolumn{3}{|l|}{0.9} & \multicolumn{3}{|l|}{0.95} \\
\hline & & & 100 & 200 & 500 & 100 & 200 & 500 & 100 & 200 & 500 & 100 & 200 & 500 \\
\hline \multirow[t]{3}{*}{2} & $\begin{array}{l}0 . \\
3\end{array}$ & & $\begin{array}{l}0.69 \\
3\end{array}$ & $\begin{array}{l}0.86 \\
5\end{array}$ & $\begin{array}{l}0.97 \\
4\end{array}$ & $\begin{array}{l}0.68 \\
2\end{array}$ & $\begin{array}{l}0.86 \\
6\end{array}$ & $\begin{array}{l}0.97 \\
6\end{array}$ & $\begin{array}{l}0.17 \\
4\end{array}$ & $\begin{array}{l}0.49 \\
5\end{array}$ & $\begin{array}{l}0.85 \\
7\end{array}$ & $\begin{array}{l}0.01 \\
4\end{array}$ & $\begin{array}{l}0.08 \\
6\end{array}$ & $\begin{array}{l}0.52 \\
4\end{array}$ \\
\hline & $\begin{array}{l}0 . \\
5\end{array}$ & & $\begin{array}{l}0.29 \\
5\end{array}$ & $\begin{array}{l}0.64 \\
5\end{array}$ & $\begin{array}{l}0.96 \\
9\end{array}$ & $\begin{array}{l}0.29 \\
6\end{array}$ & $\begin{array}{l}0.62 \\
8\end{array}$ & $\begin{array}{l}0.97 \\
1\end{array}$ & $\begin{array}{l}0.01 \\
2\end{array}$ & $\begin{array}{l}0.07 \\
0\end{array}$ & $\begin{array}{l}0.37 \\
3\end{array}$ & $\begin{array}{l}0.00 \\
3\end{array}$ & $\begin{array}{l}0.00 \\
5\end{array}$ & $\begin{array}{l}0.06 \\
5\end{array}$ \\
\hline & $\begin{array}{l}0 . \\
7\end{array}$ & & $\begin{array}{l}0.05 \\
0\end{array}$ & $\begin{array}{l}0.25 \\
2\end{array}$ & $\begin{array}{l}0.77 \\
2\end{array}$ & $\begin{array}{l}0.05 \\
9\end{array}$ & $\begin{array}{l}0.25 \\
3\end{array}$ & $\begin{array}{l}0.77 \\
0\end{array}$ & $\begin{array}{l}0.00 \\
4\end{array}$ & $\begin{array}{l}0.00 \\
6\end{array}$ & $\begin{array}{l}0.07 \\
9\end{array}$ & $\begin{array}{l}0.00 \\
2\end{array}$ & $\begin{array}{l}0.00 \\
2\end{array}$ & $\begin{array}{l}0.00 \\
6\end{array}$ \\
\hline \multirow[t]{3}{*}{3} & $\begin{array}{l}0 . \\
3\end{array}$ & & $\begin{array}{l}0.73 \\
8\end{array}$ & $\begin{array}{l}0.89 \\
7\end{array}$ & $\begin{array}{l}0.98 \\
4\end{array}$ & $\begin{array}{l}0.72 \\
6\end{array}$ & $\begin{array}{l}0.90 \\
1\end{array}$ & $\begin{array}{l}0.98 \\
5\end{array}$ & $\begin{array}{l}0.16 \\
9\end{array}$ & $\begin{array}{l}0.58 \\
8\end{array}$ & $\begin{array}{l}0.90 \\
0\end{array}$ & $\begin{array}{l}0.01 \\
4\end{array}$ & $\begin{array}{l}0.07 \\
7\end{array}$ & $\begin{array}{l}0.63 \\
2\end{array}$ \\
\hline & $\begin{array}{l}0 . \\
5\end{array}$ & & $\begin{array}{l}0.28 \\
8\end{array}$ & $\begin{array}{l}0.64 \\
4\end{array}$ & $\begin{array}{l}0.96 \\
8\end{array}$ & $\begin{array}{l}0.28 \\
4\end{array}$ & $\begin{array}{l}0.63 \\
1\end{array}$ & $\begin{array}{l}0.96 \\
8\end{array}$ & $\begin{array}{l}0.01 \\
0\end{array}$ & $\begin{array}{l}0.06 \\
3\end{array}$ & $\begin{array}{l}0.35 \\
4\end{array}$ & $\begin{array}{l}0.00 \\
5\end{array}$ & $\begin{array}{l}0.00 \\
6\end{array}$ & $\begin{array}{l}0.05 \\
8\end{array}$ \\
\hline & $\begin{array}{l}0 . \\
7\end{array}$ & & $\begin{array}{l}0.04 \\
4\end{array}$ & $\begin{array}{l}0.25 \\
5 \\
\end{array}$ & $\begin{array}{l}0.75 \\
7\end{array}$ & $\begin{array}{l}0.04 \\
8\end{array}$ & $\begin{array}{l}0.24 \\
7\end{array}$ & $\begin{array}{l}0.76 \\
9\end{array}$ & $\begin{array}{l}0.00 \\
7\end{array}$ & $\begin{array}{l}0.00 \\
7\end{array}$ & $\begin{array}{l}0.06 \\
8\end{array}$ & $\begin{array}{l}0.00 \\
4\end{array}$ & $\begin{array}{l}0.00 \\
3\end{array}$ & $\begin{array}{l}0.00 \\
3\end{array}$ \\
\hline \multirow[t]{3}{*}{4} & $\begin{array}{l}0 . \\
3\end{array}$ & & $\begin{array}{l}0.75 \\
6\end{array}$ & $\begin{array}{l}0.91 \\
4\end{array}$ & $\begin{array}{l}0.98 \\
5\end{array}$ & $\begin{array}{l}0.74 \\
9\end{array}$ & $\begin{array}{l}0.90 \\
1\end{array}$ & $\begin{array}{l}0.98 \\
8\end{array}$ & $\begin{array}{l}0.17 \\
2\end{array}$ & $\begin{array}{l}0.61 \\
8\end{array}$ & $\begin{array}{l}0.92 \\
1\end{array}$ & $\begin{array}{l}0.02 \\
1\end{array}$ & $\begin{array}{l}0.07 \\
4\end{array}$ & $\begin{array}{l}0.68 \\
0\end{array}$ \\
\hline & $\begin{array}{l}0 . \\
5\end{array}$ & & $\begin{array}{l}0.27 \\
7\end{array}$ & $\begin{array}{l}0.63 \\
5\end{array}$ & $\begin{array}{l}0.97 \\
0\end{array}$ & $\begin{array}{l}0.30 \\
4\end{array}$ & $\begin{array}{l}0.63 \\
1\end{array}$ & $\begin{array}{l}0.97 \\
0\end{array}$ & $\begin{array}{l}0.01 \\
5\end{array}$ & $\begin{array}{l}0.05 \\
0\end{array}$ & $\begin{array}{l}0.34 \\
7\end{array}$ & $\begin{array}{l}0.00 \\
5\end{array}$ & $\begin{array}{l}0.00 \\
6\end{array}$ & $\begin{array}{l}0.05 \\
4\end{array}$ \\
\hline & $\begin{array}{l}0 . \\
7\end{array}$ & & $\begin{array}{l}0.03 \\
8\end{array}$ & $\begin{array}{l}0.24 \\
6\end{array}$ & $\begin{array}{l}0.75 \\
9\end{array}$ & $\begin{array}{l}0.03 \\
6\end{array}$ & $\begin{array}{l}0.24 \\
2\end{array}$ & $\begin{array}{l}0.77 \\
4\end{array}$ & $\begin{array}{l}0.00 \\
6\end{array}$ & $\begin{array}{l}0.00 \\
7\end{array}$ & $\begin{array}{l}0.04 \\
8\end{array}$ & $\begin{array}{l}0.00 \\
4\end{array}$ & $\begin{array}{l}0.00 \\
2\end{array}$ & $\begin{array}{l}0.00 \\
3\end{array}$ \\
\hline \multirow[t]{3}{*}{$1 / 2$} & $\begin{array}{l}0 . \\
3\end{array}$ & & $\begin{array}{l}0.32 \\
4\end{array}$ & $\begin{array}{l}0.50 \\
5\end{array}$ & $\begin{array}{l}0.72 \\
4\end{array}$ & $\begin{array}{l}0.26 \\
6\end{array}$ & $\begin{array}{l}0.46 \\
6\end{array}$ & $\begin{array}{l}0.76 \\
4\end{array}$ & $\begin{array}{l}0.13 \\
6\end{array}$ & $\begin{array}{l}0.23 \\
2\end{array}$ & $\begin{array}{l}0.60 \\
1\end{array}$ & $\begin{array}{l}0.06 \\
7\end{array}$ & $\begin{array}{l}0.09 \\
7\end{array}$ & $\begin{array}{l}0.21 \\
6\end{array}$ \\
\hline & $\begin{array}{l}0 . \\
5\end{array}$ & & $\begin{array}{l}0.16 \\
0\end{array}$ & $\begin{array}{l}0.38 \\
7\end{array}$ & $\begin{array}{l}0.74 \\
1\end{array}$ & $\begin{array}{l}0.09 \\
9\end{array}$ & $\begin{array}{l}0.26 \\
0\end{array}$ & $\begin{array}{l}0.67 \\
3\end{array}$ & $\begin{array}{l}0.02 \\
7\end{array}$ & $\begin{array}{l}0.07 \\
0\end{array}$ & $\begin{array}{l}0.31 \\
0\end{array}$ & $\begin{array}{l}0.00 \\
8\end{array}$ & $\begin{array}{l}0.01 \\
8\end{array}$ & $\begin{array}{l}0.06 \\
7\end{array}$ \\
\hline & $\begin{array}{l}0 . \\
7\end{array}$ & & $\begin{array}{l}0.02 \\
6\end{array}$ & $\begin{array}{l}0.11 \\
9 \\
\end{array}$ & $\begin{array}{l}0.53 \\
9\end{array}$ & $\begin{array}{l}0.00 \\
6\end{array}$ & $\begin{array}{l}0.02 \\
8\end{array}$ & $\begin{array}{l}0.27 \\
8\end{array}$ & $\begin{array}{l}0.00 \\
0\end{array}$ & $\begin{array}{l}0.00 \\
5\end{array}$ & $\begin{array}{l}0.03 \\
7 \\
\end{array}$ & $\begin{array}{l}0.00 \\
0\end{array}$ & $\begin{array}{l}0.00 \\
0\end{array}$ & $\begin{array}{l}0.00 \\
3 \\
\end{array}$ \\
\hline \multirow[t]{3}{*}{$1 / 3$} & $\begin{array}{l}0 . \\
3\end{array}$ & & $\begin{array}{l}0.08 \\
2\end{array}$ & $\begin{array}{l}0.20 \\
6\end{array}$ & $\begin{array}{l}0.42 \\
0\end{array}$ & $\begin{array}{l}0.13 \\
8\end{array}$ & $\begin{array}{l}0.26 \\
9\end{array}$ & $\begin{array}{l}0.54 \\
8\end{array}$ & $\begin{array}{l}0.11 \\
7\end{array}$ & $\begin{array}{l}0.18 \\
9\end{array}$ & $\begin{array}{l}0.49 \\
9\end{array}$ & $\begin{array}{l}0.07 \\
6\end{array}$ & $\begin{array}{l}0.09 \\
8\end{array}$ & $\begin{array}{l}0.20 \\
6\end{array}$ \\
\hline & $\begin{array}{l}0 . \\
5\end{array}$ & & $\begin{array}{l}0.04 \\
1\end{array}$ & $\begin{array}{l}0.15 \\
4\end{array}$ & $\begin{array}{l}0.42 \\
1\end{array}$ & $\begin{array}{l}0.03 \\
2\end{array}$ & $\begin{array}{l}0.10 \\
7\end{array}$ & $\begin{array}{l}0.42 \\
3\end{array}$ & $\begin{array}{l}0.01 \\
2\end{array}$ & $\begin{array}{l}0.02 \\
9\end{array}$ & $\begin{array}{l}0.15 \\
3\end{array}$ & $\begin{array}{l}0.00 \\
2\end{array}$ & $\begin{array}{l}0.00 \\
8\end{array}$ & $\begin{array}{l}0.02 \\
8\end{array}$ \\
\hline & $\begin{array}{l}0 . \\
7\end{array}$ & & $\begin{array}{l}0.00 \\
6\end{array}$ & $\begin{array}{l}0.03 \\
0\end{array}$ & $\begin{array}{l}0.23 \\
5\end{array}$ & $\begin{array}{l}0.00 \\
2\end{array}$ & $\begin{array}{l}0.00 \\
7\end{array}$ & $\begin{array}{l}0.09 \\
3\end{array}$ & $\begin{array}{l}0.00 \\
0\end{array}$ & $\begin{array}{l}0.00 \\
0\end{array}$ & $\begin{array}{l}0.00 \\
9\end{array}$ & $\begin{array}{l}0.00 \\
0\end{array}$ & $\begin{array}{l}0.00 \\
0\end{array}$ & $\begin{array}{l}0.00 \\
0\end{array}$ \\
\hline \multirow[t]{3}{*}{$1 / 4$} & $\begin{array}{l}0 . \\
3\end{array}$ & & $\begin{array}{l}0.00 \\
9\end{array}$ & $\begin{array}{l}0.03 \\
5\end{array}$ & $\begin{array}{l}0.15 \\
1\end{array}$ & $\begin{array}{l}0.03 \\
8\end{array}$ & $\begin{array}{l}0.09 \\
2\end{array}$ & $\begin{array}{l}0.27 \\
5\end{array}$ & $\begin{array}{l}0.07 \\
3\end{array}$ & $\begin{array}{l}0.12 \\
7\end{array}$ & $\begin{array}{l}0.37 \\
1\end{array}$ & $\begin{array}{l}0.06 \\
7\end{array}$ & $\begin{array}{l}0.07 \\
4\end{array}$ & $\begin{array}{l}0.17 \\
2\end{array}$ \\
\hline & $\begin{array}{l}0 . \\
5\end{array}$ & & $\begin{array}{l}0.00 \\
7\end{array}$ & $\begin{array}{l}0.02 \\
4\end{array}$ & $\begin{array}{l}0.16 \\
1\end{array}$ & $\begin{array}{l}0.00 \\
5\end{array}$ & $\begin{array}{l}0.02 \\
6\end{array}$ & $\begin{array}{l}0.19 \\
6\end{array}$ & $\begin{array}{l}0.00 \\
2\end{array}$ & $\begin{array}{l}0.00 \\
7\end{array}$ & $\begin{array}{l}0.05 \\
2\end{array}$ & $\begin{array}{l}0.00 \\
1\end{array}$ & $\begin{array}{l}0.00 \\
1\end{array}$ & $\begin{array}{l}0.00 \\
6\end{array}$ \\
\hline & $\begin{array}{l}0 . \\
7\end{array}$ & & $\begin{array}{l}0.00 \\
1\end{array}$ & $\begin{array}{l}0.00 \\
6\end{array}$ & $\begin{array}{l}0.08 \\
1\end{array}$ & $\begin{array}{l}0.00 \\
0\end{array}$ & $\begin{array}{l}0.00 \\
0\end{array}$ & $\begin{array}{l}0.02 \\
5\end{array}$ & $\begin{array}{l}0.00 \\
0\end{array}$ & $\begin{array}{l}0.00 \\
0\end{array}$ & $\begin{array}{l}0.00 \\
2\end{array}$ & $\begin{array}{l}0.00 \\
0\end{array}$ & $\begin{array}{l}0.00 \\
0\end{array}$ & $\begin{array}{l}0.00 \\
0\end{array}$ \\
\hline
\end{tabular}

Table 5:- Power under $H_{01}$ with a time-varying variance change

\begin{tabular}{|l|l|l|l|l|l|l|}
\hline & Table 5:- Power under $H_{01}$ with a time-varying variance change & 0.9 & 0.95 \\
\hline
\end{tabular}




\begin{tabular}{|c|c|c|c|c|c|c|c|c|c|c|c|c|c|c|}
\hline$\sigma_{1} / \sigma_{2}$ & $\lambda$ & $T$ & 100 & 200 & 500 & 100 & 200 & 500 & 100 & 200 & 500 & 100 & 200 & 500 \\
\hline \multirow[t]{3}{*}{2} & $\begin{array}{l}0 . \\
3\end{array}$ & & $\begin{array}{l}0.86 \\
9\end{array}$ & $\begin{array}{l}0.94 \\
7\end{array}$ & $\begin{array}{l}0.99 \\
3\end{array}$ & $\begin{array}{l}0.83 \\
9\end{array}$ & $\begin{array}{l}0.94 \\
4\end{array}$ & $\begin{array}{l}0.98 \\
2\end{array}$ & $\begin{array}{l}0.77 \\
2\end{array}$ & $\begin{array}{l}0.90 \\
9\end{array}$ & $\begin{array}{l}0.97 \\
8\end{array}$ & $\begin{array}{l}0.68 \\
3\end{array}$ & $\begin{array}{l}0.82 \\
9\end{array}$ & $\begin{array}{l}0.95 \\
5\end{array}$ \\
\hline & $\begin{array}{l}0 . \\
5\end{array}$ & & $\begin{array}{l}0.91 \\
7\end{array}$ & $\begin{array}{l}0.97 \\
3\end{array}$ & $\begin{array}{l}0.99 \\
6\end{array}$ & $\begin{array}{l}0.91 \\
2\end{array}$ & $\begin{array}{l}0.96 \\
6\end{array}$ & $\begin{array}{l}0.98 \\
9\end{array}$ & $\begin{array}{l}0.88 \\
9\end{array}$ & $\begin{array}{l}0.95 \\
7\end{array}$ & $\begin{array}{l}0.98 \\
7\end{array}$ & $\begin{array}{l}0.81 \\
1\end{array}$ & $\begin{array}{l}0.91 \\
4\end{array}$ & $\begin{array}{l}0.97 \\
5\end{array}$ \\
\hline & $\begin{array}{l}0 . \\
7 \\
\end{array}$ & & $\begin{array}{l}0.86 \\
3 \\
\end{array}$ & $\begin{array}{l}0.92 \\
5 \\
\end{array}$ & $\begin{array}{l}0.98 \\
2\end{array}$ & $\begin{array}{l}0.86 \\
3 \\
\end{array}$ & $\begin{array}{l}0.92 \\
7 \\
\end{array}$ & $\begin{array}{l}0.97 \\
3\end{array}$ & $\begin{array}{l}0.86 \\
5\end{array}$ & $\begin{array}{l}0.93 \\
1 \\
\end{array}$ & $\begin{array}{l}0.97 \\
1\end{array}$ & $\begin{array}{l}0.82 \\
7\end{array}$ & $\begin{array}{l}0.91 \\
1 \\
\end{array}$ & $\begin{array}{l}0.96 \\
5\end{array}$ \\
\hline \multirow[t]{3}{*}{3} & $\begin{array}{l}0 . \\
3\end{array}$ & & $\begin{array}{l}0.89 \\
3\end{array}$ & $\begin{array}{l}0.95 \\
5\end{array}$ & $\begin{array}{l}0.99 \\
2\end{array}$ & $\begin{array}{l}0.84 \\
7\end{array}$ & $\begin{array}{l}0.95 \\
1\end{array}$ & $\begin{array}{l}0.98 \\
6\end{array}$ & $\begin{array}{l}0.77 \\
7\end{array}$ & $\begin{array}{l}0.91 \\
7\end{array}$ & $\begin{array}{l}0.98 \\
1\end{array}$ & $\begin{array}{l}0.70 \\
3\end{array}$ & $\begin{array}{l}0.84 \\
3\end{array}$ & $\begin{array}{l}0.96 \\
7\end{array}$ \\
\hline & $\begin{array}{l}0 . \\
5\end{array}$ & & $\begin{array}{l}0.93 \\
0\end{array}$ & $\begin{array}{l}0.97 \\
0\end{array}$ & $\begin{array}{l}0.99 \\
5\end{array}$ & $\begin{array}{l}0.92 \\
1\end{array}$ & $\begin{array}{l}0.96 \\
1\end{array}$ & $\begin{array}{l}0.99 \\
1\end{array}$ & $\begin{array}{l}0.89 \\
3\end{array}$ & $\begin{array}{l}0.96 \\
0\end{array}$ & $\begin{array}{l}0.98 \\
1\end{array}$ & $\begin{array}{l}0.82 \\
8\end{array}$ & $\begin{array}{l}0.93 \\
7\end{array}$ & $\begin{array}{l}0.98 \\
2\end{array}$ \\
\hline & $\begin{array}{l}0 . \\
7 \\
\end{array}$ & & $\begin{array}{l}0.84 \\
8 \\
\end{array}$ & $\begin{array}{l}0.91 \\
3\end{array}$ & $\begin{array}{l}0.97 \\
3 \\
\end{array}$ & $\begin{array}{l}0.84 \\
2\end{array}$ & $\begin{array}{l}0.92 \\
2\end{array}$ & $\begin{array}{l}0.97 \\
0 \\
\end{array}$ & $\begin{array}{l}0.84 \\
7 \\
\end{array}$ & $\begin{array}{l}0.91 \\
9\end{array}$ & $\begin{array}{l}0.96 \\
8 \\
\end{array}$ & $\begin{array}{l}0.82 \\
2 \\
\end{array}$ & $\begin{array}{l}0.91 \\
9\end{array}$ & $\begin{array}{l}0.96 \\
6 \\
\end{array}$ \\
\hline \multirow[t]{3}{*}{4} & $\begin{array}{l}0 . \\
3\end{array}$ & & $\begin{array}{l}0.90 \\
0\end{array}$ & $\begin{array}{l}0.95 \\
5\end{array}$ & $\begin{array}{l}0.99 \\
1\end{array}$ & $\begin{array}{l}0.86 \\
0\end{array}$ & $\begin{array}{l}0.95 \\
1\end{array}$ & $\begin{array}{l}0.98 \\
6\end{array}$ & $\begin{array}{l}0.78 \\
6\end{array}$ & $\begin{array}{l}0.92 \\
0\end{array}$ & $\begin{array}{l}0.97 \\
8\end{array}$ & $\begin{array}{l}0.69 \\
7\end{array}$ & $\begin{array}{l}0.85 \\
5\end{array}$ & $\begin{array}{l}0.97 \\
6\end{array}$ \\
\hline & $\begin{array}{l}0 . \\
5\end{array}$ & & $\begin{array}{l}0.93 \\
7\end{array}$ & $\begin{array}{l}0.96 \\
9\end{array}$ & $\begin{array}{l}0.99 \\
4\end{array}$ & $\begin{array}{l}0.92 \\
5\end{array}$ & $\begin{array}{l}0.96 \\
3\end{array}$ & $\begin{array}{l}0.99 \\
0\end{array}$ & $\begin{array}{l}0.89 \\
9\end{array}$ & $\begin{array}{l}0.95 \\
8\end{array}$ & $\begin{array}{l}0.98 \\
7\end{array}$ & $\begin{array}{l}0.83 \\
2\end{array}$ & $\begin{array}{l}0.93 \\
6\end{array}$ & $\begin{array}{l}0.98 \\
4\end{array}$ \\
\hline & $\begin{array}{l}0 . \\
7\end{array}$ & & $\begin{array}{l}0.82 \\
3\end{array}$ & $\begin{array}{l}0.90 \\
6\end{array}$ & $\begin{array}{l}0.96 \\
7\end{array}$ & $\begin{array}{l}0.82 \\
9\end{array}$ & $\begin{array}{l}0.91 \\
3\end{array}$ & $\begin{array}{l}0.96 \\
6\end{array}$ & $\begin{array}{l}0.85 \\
1\end{array}$ & $\begin{array}{l}0.91 \\
7\end{array}$ & $\begin{array}{l}0.96 \\
4\end{array}$ & $\begin{array}{l}0.82 \\
9\end{array}$ & $\begin{array}{l}0.92 \\
3\end{array}$ & $\begin{array}{l}0.96 \\
5\end{array}$ \\
\hline \multirow[t]{3}{*}{$1 / 2$} & $\begin{array}{l}0 . \\
3\end{array}$ & & $\begin{array}{l}0.54 \\
5\end{array}$ & $\begin{array}{l}0.82 \\
4\end{array}$ & $\begin{array}{l}0.98 \\
0\end{array}$ & $\begin{array}{l}0.49 \\
0\end{array}$ & $\begin{array}{l}0.76 \\
5\end{array}$ & $\begin{array}{l}0.95 \\
4\end{array}$ & $\begin{array}{l}0.47 \\
0\end{array}$ & $\begin{array}{l}0.68 \\
9\end{array}$ & $\begin{array}{l}0.91 \\
0\end{array}$ & $\begin{array}{l}0.47 \\
4\end{array}$ & $\begin{array}{l}0.66 \\
2\end{array}$ & $\begin{array}{l}0.86 \\
9\end{array}$ \\
\hline & $\begin{array}{l}0 . \\
5\end{array}$ & & $\begin{array}{l}0.72 \\
3\end{array}$ & $\begin{array}{l}0.92 \\
3\end{array}$ & $\begin{array}{l}0.99 \\
5\end{array}$ & $\begin{array}{l}0.65 \\
9\end{array}$ & $\begin{array}{l}0.87 \\
9\end{array}$ & $\begin{array}{l}0.99 \\
2\end{array}$ & $\begin{array}{l}0.60 \\
4\end{array}$ & $\begin{array}{l}0.81 \\
5\end{array}$ & $\begin{array}{l}0.95 \\
7\end{array}$ & $\begin{array}{l}0.62 \\
6\end{array}$ & $\begin{array}{l}0.78 \\
3\end{array}$ & $\begin{array}{l}0.92 \\
4\end{array}$ \\
\hline & $\begin{array}{l}0 . \\
7 \\
\end{array}$ & & $\begin{array}{l}0.88 \\
3 \\
\end{array}$ & $\begin{array}{l}0.96 \\
8\end{array}$ & $\begin{array}{l}0.99 \\
8\end{array}$ & $\begin{array}{l}0.86 \\
1\end{array}$ & $\begin{array}{l}0.95 \\
4 \\
\end{array}$ & $\begin{array}{l}0.99 \\
5\end{array}$ & $\begin{array}{l}0.82 \\
8\end{array}$ & $\begin{array}{l}0.91 \\
9 \\
\end{array}$ & $\begin{array}{l}0.98 \\
3 \\
\end{array}$ & $\begin{array}{l}0.82 \\
1\end{array}$ & $\begin{array}{l}0.91 \\
8 \\
\end{array}$ & $\begin{array}{l}0.96 \\
9\end{array}$ \\
\hline \multirow[t]{3}{*}{$1 / 3$} & $\begin{array}{l}0 . \\
3\end{array}$ & & $\begin{array}{l}0.40 \\
6\end{array}$ & $\begin{array}{l}0.69 \\
2\end{array}$ & $\begin{array}{l}0.96 \\
2\end{array}$ & $\begin{array}{l}0.35 \\
3\end{array}$ & $\begin{array}{l}0.60 \\
2\end{array}$ & $\begin{array}{l}0.90 \\
5\end{array}$ & $\begin{array}{l}0.30 \\
4\end{array}$ & $\begin{array}{l}0.51 \\
6\end{array}$ & $\begin{array}{l}0.81 \\
7\end{array}$ & $\begin{array}{l}0.32 \\
0\end{array}$ & $\begin{array}{l}0.51 \\
6\end{array}$ & $\begin{array}{l}0.76 \\
2\end{array}$ \\
\hline & $\begin{array}{l}0 . \\
5\end{array}$ & & $\begin{array}{l}0.60 \\
1\end{array}$ & $\begin{array}{l}0.86 \\
6\end{array}$ & $\begin{array}{l}0.99 \\
5\end{array}$ & & $\begin{array}{l}0.77 \\
7\end{array}$ & $\begin{array}{l}0.97 \\
7\end{array}$ & $\begin{array}{l}0.44 \\
0\end{array}$ & $\begin{array}{l}0.67 \\
5\end{array}$ & $\begin{array}{l}0.91 \\
3\end{array}$ & $\begin{array}{l}0.45 \\
0\end{array}$ & $\begin{array}{l}0.62 \\
6\end{array}$ & $\begin{array}{l}0.84 \\
7\end{array}$ \\
\hline & $\begin{array}{l}0 . \\
7\end{array}$ & & $\begin{array}{l}0.89 \\
3\end{array}$ & $\begin{array}{l}0.97 \\
1\end{array}$ & $\begin{array}{l}0.99 \\
8\end{array}$ & $\begin{array}{l}0.86 \\
1\end{array}$ & $\begin{array}{l}0.95 \\
6\end{array}$ & $\begin{array}{l}0.99 \\
5\end{array}$ & $\begin{array}{l}0.82 \\
0\end{array}$ & $\begin{array}{l}0.93 \\
1\end{array}$ & $\begin{array}{l}0.98 \\
6\end{array}$ & $\begin{array}{l}0.79 \\
2\end{array}$ & $\begin{array}{l}0.91 \\
0\end{array}$ & $\begin{array}{l}0.97 \\
2\end{array}$ \\
\hline \multirow[t]{3}{*}{$1 / 4$} & $\begin{array}{l}0 . \\
3\end{array}$ & & $\begin{array}{l}0.35 \\
5\end{array}$ & $\begin{array}{l}0.61 \\
5\end{array}$ & $\begin{array}{l}0.94 \\
7\end{array}$ & $\begin{array}{l}0.29 \\
2\end{array}$ & $\begin{array}{l}0.50 \\
4\end{array}$ & $\begin{array}{l}0.87 \\
0\end{array}$ & $\begin{array}{l}0.24 \\
5\end{array}$ & $\begin{array}{l}0.41 \\
3\end{array}$ & $\begin{array}{l}0.73 \\
3\end{array}$ & $\begin{array}{l}0.23 \\
7\end{array}$ & $\begin{array}{l}0.38 \\
2\end{array}$ & $\begin{array}{l}0.63 \\
6\end{array}$ \\
\hline & $\begin{array}{l}0 . \\
5\end{array}$ & & $\begin{array}{l}0.54 \\
3\end{array}$ & $\begin{array}{l}0.81 \\
8\end{array}$ & $\begin{array}{l}0.99 \\
0\end{array}$ & $\begin{array}{l}0.45 \\
1\end{array}$ & $\begin{array}{l}0.70 \\
2\end{array}$ & $\begin{array}{l}0.95 \\
9\end{array}$ & $\begin{array}{l}0.36 \\
1\end{array}$ & $\begin{array}{l}0.58 \\
0\end{array}$ & $\begin{array}{l}0.86 \\
0\end{array}$ & $\begin{array}{l}0.34 \\
8\end{array}$ & $\begin{array}{l}0.49 \\
5\end{array}$ & $\begin{array}{l}0.77 \\
1\end{array}$ \\
\hline & $\begin{array}{l}0 . \\
7\end{array}$ & & $\begin{array}{l}0.89 \\
0\end{array}$ & $\begin{array}{l}0.97 \\
6\end{array}$ & $\begin{array}{l}0.99 \\
8\end{array}$ & $\begin{array}{l}0.86 \\
8\end{array}$ & $\begin{array}{l}0.96 \\
0\end{array}$ & $\begin{array}{l}0.99 \\
7\end{array}$ & $\begin{array}{l}0.82 \\
9\end{array}$ & $\begin{array}{l}0.93 \\
6\end{array}$ & $\begin{array}{l}0.98 \\
6\end{array}$ & $\begin{array}{l}0.79 \\
5\end{array}$ & $\begin{array}{l}0.91 \\
9\end{array}$ & $\begin{array}{l}0.97 \\
9\end{array}$ \\
\hline
\end{tabular}

Table 6:- Power under $H_{01}$ with an abrupt variance change

\begin{tabular}{|c|c|c|c|c|c|c|c|c|c|c|c|c|c|c|}
\hline \multirow{2}{*}{$\begin{array}{l}\sigma_{1} / \sigma_{2} \\
\end{array}$} & \multirow[b]{2}{*}{$\lambda$} & \multirow{2}{*}{$\begin{array}{l}\rho \\
T\end{array}$} & \multicolumn{3}{|l|}{0.7} & \multicolumn{3}{|l|}{0.8} & \multicolumn{3}{|l|}{0.9} & \multicolumn{3}{|l|}{0.95} \\
\hline & & & 100 & 200 & 500 & 100 & 200 & 500 & 100 & 200 & 500 & 100 & 200 & 500 \\
\hline \multirow[t]{3}{*}{2} & $\begin{array}{l}0 . \\
3\end{array}$ & & $\begin{array}{l}0.91 \\
1\end{array}$ & $\begin{array}{l}0.96 \\
8\end{array}$ & $\begin{array}{l}0.99 \\
3\end{array}$ & $\begin{array}{l}0.87 \\
7\end{array}$ & $\begin{array}{l}0.96 \\
0\end{array}$ & $\begin{array}{l}0.98 \\
7\end{array}$ & $\begin{array}{l}0.82 \\
4\end{array}$ & $\begin{array}{l}0.93 \\
8\end{array}$ & $\begin{array}{l}0.98 \\
3\end{array}$ & $\begin{array}{l}0.79 \\
1\end{array}$ & $\begin{array}{l}0.88 \\
5\end{array}$ & $\begin{array}{l}0.97 \\
8\end{array}$ \\
\hline & $\begin{array}{l}0 . \\
5\end{array}$ & & $\begin{array}{l}0.94 \\
4\end{array}$ & $\begin{array}{l}0.97 \\
7\end{array}$ & $\begin{array}{l}0.99 \\
2\end{array}$ & $\begin{array}{l}0.94 \\
3\end{array}$ & $\begin{array}{l}0.97 \\
0\end{array}$ & $\begin{array}{l}0.99 \\
2\end{array}$ & $\begin{array}{l}0.91 \\
4\end{array}$ & $\begin{array}{l}0.97 \\
2\end{array}$ & $\begin{array}{l}0.98 \\
7\end{array}$ & $\begin{array}{l}0.89 \\
8\end{array}$ & $\begin{array}{l}0.95 \\
9\end{array}$ & $\begin{array}{l}0.98 \\
7\end{array}$ \\
\hline & 0. & & 0.70 & 0.85 & 0.94 & 0.69 & 0.84 & 0.94 & 0.72 & 0.83 & 0.93 & 0.80 & 0.85 & 0.93 \\
\hline
\end{tabular}




\begin{tabular}{|c|c|c|c|c|c|c|c|c|c|c|c|c|c|}
\hline & 7 & 0 & 5 & 6 & 0 & 9 & 3 & 6 & 5 & 7 & 5 & 5 & 7 \\
\hline \multirow{4}{*}{3} & & & & & & & & & & & & & \\
\hline & $\begin{array}{l}0 . \\
3\end{array}$ & $\begin{array}{l}0.91 \\
5\end{array}$ & $\begin{array}{l}0.96 \\
9\end{array}$ & $\begin{array}{l}0.99 \\
2\end{array}$ & $\begin{array}{l}0.88 \\
1\end{array}$ & $\begin{array}{l}0.96 \\
1\end{array}$ & $\begin{array}{l}0.98 \\
8\end{array}$ & $\begin{array}{l}0.81 \\
8\end{array}$ & $\begin{array}{l}0.93 \\
9\end{array}$ & $\begin{array}{l}0.98 \\
7 \\
\end{array}$ & $\begin{array}{l}0.79 \\
3 \\
\end{array}$ & $\begin{array}{l}0.88 \\
8\end{array}$ & $\begin{array}{l}0.97 \\
7\end{array}$ \\
\hline & $\begin{array}{l}0 . \\
5\end{array}$ & $\begin{array}{l}0.94 \\
4\end{array}$ & $\begin{array}{l}0.97 \\
8\end{array}$ & $\begin{array}{l}0.99 \\
0\end{array}$ & $\begin{array}{l}0.94 \\
2\end{array}$ & $\begin{array}{l}0.97 \\
5\end{array}$ & $\begin{array}{l}0.99 \\
0\end{array}$ & $\begin{array}{l}0.92 \\
1\end{array}$ & $\begin{array}{l}0.97 \\
4\end{array}$ & $\begin{array}{l}0.98 \\
9\end{array}$ & $\begin{array}{l}0.90 \\
1\end{array}$ & $\begin{array}{l}0.95 \\
8\end{array}$ & $\begin{array}{l}0.99 \\
0\end{array}$ \\
\hline & $\begin{array}{l}0 . \\
7 \\
\end{array}$ & $\begin{array}{l}0.40 \\
5 \\
\end{array}$ & $\begin{array}{l}0.67 \\
4 \\
\end{array}$ & $\begin{array}{l}0.86 \\
3 \\
\end{array}$ & $\begin{array}{l}0.39 \\
6 \\
\end{array}$ & $\begin{array}{l}0.66 \\
1 \\
\end{array}$ & $\begin{array}{l}0.86 \\
4 \\
\end{array}$ & $\begin{array}{l}0.55 \\
2 \\
\end{array}$ & $\begin{array}{l}0.66 \\
0 \\
\end{array}$ & $\begin{array}{l}0.85 \\
7 \\
\end{array}$ & $\begin{array}{l}0.79 \\
5 \\
\end{array}$ & $\begin{array}{l}0.76 \\
0 \\
\end{array}$ & $\begin{array}{l}0.85 \\
4 \\
\end{array}$ \\
\hline \multirow[t]{3}{*}{4} & $\begin{array}{l}0 . \\
3\end{array}$ & $\begin{array}{l}0.92 \\
3\end{array}$ & $\begin{array}{l}0.96 \\
2\end{array}$ & $\begin{array}{l}0.99 \\
0\end{array}$ & $\begin{array}{l}0.88 \\
6\end{array}$ & $\begin{array}{l}0.95 \\
9\end{array}$ & $\begin{array}{l}0.98 \\
9\end{array}$ & $\begin{array}{l}0.83 \\
5\end{array}$ & $\begin{array}{l}0.94 \\
1\end{array}$ & $\begin{array}{l}0.98 \\
4\end{array}$ & $\begin{array}{l}0.79 \\
4\end{array}$ & $\begin{array}{l}0.88 \\
9\end{array}$ & $\begin{array}{l}0.97 \\
9\end{array}$ \\
\hline & $\begin{array}{l}0 . \\
5\end{array}$ & $\begin{array}{l}0.95 \\
0\end{array}$ & $\begin{array}{l}0.97 \\
0\end{array}$ & $\begin{array}{l}0.98 \\
9\end{array}$ & $\begin{array}{l}0.94 \\
2\end{array}$ & $\begin{array}{l}0.97 \\
3\end{array}$ & $\begin{array}{l}0.99 \\
0\end{array}$ & $\begin{array}{l}0.92 \\
6\end{array}$ & $\begin{array}{l}0.97 \\
1\end{array}$ & $\begin{array}{l}0.99 \\
0\end{array}$ & $\begin{array}{l}0.90 \\
4\end{array}$ & $\begin{array}{l}0.95 \\
8\end{array}$ & $\begin{array}{l}0.98 \\
4\end{array}$ \\
\hline & $\begin{array}{l}0 . \\
7\end{array}$ & $\begin{array}{l}0.15 \\
9\end{array}$ & $\begin{array}{l}0.45 \\
3\end{array}$ & $\begin{array}{l}0.74 \\
8\end{array}$ & $\begin{array}{l}0.16 \\
8\end{array}$ & $\begin{array}{l}0.44 \\
3\end{array}$ & $\begin{array}{l}0.76 \\
5\end{array}$ & $\begin{array}{l}0.45 \\
4\end{array}$ & $\begin{array}{l}0.44 \\
5\end{array}$ & $\begin{array}{l}0.74 \\
7\end{array}$ & $\begin{array}{l}0.79 \\
9\end{array}$ & $\begin{array}{l}0.72 \\
6\end{array}$ & $\begin{array}{l}0.74 \\
8\end{array}$ \\
\hline \multirow[t]{3}{*}{$1 / 2$} & $\begin{array}{l}0 . \\
3\end{array}$ & $\begin{array}{l}0.41 \\
7 \\
\end{array}$ & $\begin{array}{l}0.73 \\
7 \\
\end{array}$ & $\begin{array}{l}0.96 \\
2\end{array}$ & $\begin{array}{l}0.37 \\
0\end{array}$ & $\begin{array}{l}0.66 \\
7 \\
\end{array}$ & $\begin{array}{l}0.92 \\
7 \\
\end{array}$ & $\begin{array}{l}0.35 \\
2 \\
\end{array}$ & $\begin{array}{l}0.56 \\
8 \\
\end{array}$ & $\begin{array}{l}0.85 \\
5 \\
\end{array}$ & $\begin{array}{l}0.37 \\
2 \\
\end{array}$ & $\begin{array}{l}0.55 \\
7 \\
\end{array}$ & $\begin{array}{l}0.80 \\
8\end{array}$ \\
\hline & $\begin{array}{l}0 . \\
5\end{array}$ & $\begin{array}{l}0.68 \\
5\end{array}$ & $\begin{array}{l}0.91 \\
0\end{array}$ & $\begin{array}{l}0.99 \\
7\end{array}$ & $\begin{array}{l}0.64 \\
4\end{array}$ & $\begin{array}{l}0.85 \\
8\end{array}$ & $\begin{array}{l}0.98 \\
5\end{array}$ & $\begin{array}{l}0.59 \\
2\end{array}$ & $\begin{array}{l}0.80 \\
8\end{array}$ & $\begin{array}{l}0.95 \\
3\end{array}$ & $\begin{array}{l}0.62 \\
3\end{array}$ & $\begin{array}{l}0.78 \\
9\end{array}$ & $\begin{array}{l}0.92 \\
4\end{array}$ \\
\hline & $\begin{array}{l}0 . \\
7\end{array}$ & $\begin{array}{l}0.93 \\
2 \\
\end{array}$ & $\begin{array}{l}0.97 \\
8 \\
\end{array}$ & $\begin{array}{l}0.99 \\
8 \\
\end{array}$ & $\begin{array}{l}0.92 \\
1\end{array}$ & $\begin{array}{l}0.97 \\
2\end{array}$ & $\begin{array}{l}0.99 \\
4 \\
\end{array}$ & $\begin{array}{l}0.91 \\
2 \\
\end{array}$ & $\begin{array}{l}0.96 \\
1\end{array}$ & $\begin{array}{l}0.99 \\
1 \\
\end{array}$ & $\begin{array}{l}0.92 \\
6 \\
\end{array}$ & $\begin{array}{l}0.96 \\
0\end{array}$ & $\begin{array}{l}0.98 \\
6 \\
\end{array}$ \\
\hline \multirow[t]{3}{*}{$1 / 3$} & $\begin{array}{l}0 . \\
3\end{array}$ & $\begin{array}{l}0.30 \\
9\end{array}$ & $\begin{array}{l}0.55 \\
3 \\
\end{array}$ & $\begin{array}{l}0.93 \\
1\end{array}$ & $\begin{array}{l}0.25 \\
0\end{array}$ & $\begin{array}{l}0.46 \\
7\end{array}$ & $\begin{array}{l}0.84 \\
0\end{array}$ & $\begin{array}{l}0.21 \\
7\end{array}$ & $\begin{array}{l}0.35 \\
6\end{array}$ & $\begin{array}{l}0.69 \\
8\end{array}$ & $\begin{array}{l}0.20 \\
6\end{array}$ & $\begin{array}{l}0.34 \\
7 \\
\end{array}$ & $\begin{array}{l}0.63 \\
1\end{array}$ \\
\hline & $\begin{array}{l}0 . \\
5\end{array}$ & $\begin{array}{l}0.64 \\
9\end{array}$ & $\begin{array}{l}0.89 \\
4\end{array}$ & $\begin{array}{l}0.99 \\
6\end{array}$ & $\begin{array}{l}0.61 \\
7\end{array}$ & $\begin{array}{l}0.84 \\
1\end{array}$ & $\begin{array}{l}0.98 \\
8\end{array}$ & $\begin{array}{l}0.56 \\
6\end{array}$ & $\begin{array}{l}0.77 \\
5\end{array}$ & $\begin{array}{l}0.95 \\
4\end{array}$ & $\begin{array}{l}0.57 \\
7\end{array}$ & $\begin{array}{l}0.76 \\
5\end{array}$ & $\begin{array}{l}0.91 \\
9\end{array}$ \\
\hline & $\begin{array}{l}0 . \\
7 \\
\end{array}$ & $\begin{array}{l}0.94 \\
5 \\
\end{array}$ & $\begin{array}{l}0.98 \\
6 \\
\end{array}$ & $\begin{array}{l}0.99 \\
8 \\
\end{array}$ & $\begin{array}{l}0.94 \\
1 \\
\end{array}$ & $\begin{array}{l}0.98 \\
3 \\
\end{array}$ & $\begin{array}{l}0.99 \\
8 \\
\end{array}$ & $\begin{array}{l}0.93 \\
6 \\
\end{array}$ & $\begin{array}{l}0.97 \\
4 \\
\end{array}$ & $\begin{array}{l}0.99 \\
6 \\
\end{array}$ & $\begin{array}{l}0.94 \\
8 \\
\end{array}$ & $\begin{array}{l}0.97 \\
7 \\
\end{array}$ & $\begin{array}{l}0.99 \\
4 \\
\end{array}$ \\
\hline \multirow[t]{3}{*}{$1 / 4$} & $\begin{array}{l}0 . \\
3\end{array}$ & $\begin{array}{l}0.31 \\
0\end{array}$ & $\begin{array}{l}0.50 \\
4\end{array}$ & $\begin{array}{l}0.89 \\
4 \\
\end{array}$ & $\begin{array}{l}0.25 \\
9 \\
\end{array}$ & $\begin{array}{l}0.38 \\
9\end{array}$ & $\begin{array}{l}0.77 \\
0 \\
\end{array}$ & $\begin{array}{l}0.21 \\
6 \\
\end{array}$ & $\begin{array}{l}0.29 \\
5 \\
\end{array}$ & $\begin{array}{l}0.59 \\
1 \\
\end{array}$ & $\begin{array}{l}0.17 \\
5 \\
\end{array}$ & $\begin{array}{l}0.25 \\
2\end{array}$ & $\begin{array}{l}0.48 \\
1 \\
\end{array}$ \\
\hline & $\begin{array}{l}0 . \\
5\end{array}$ & $\begin{array}{l}0.67 \\
1\end{array}$ & $\begin{array}{l}0.90 \\
4\end{array}$ & $\begin{array}{l}0.99 \\
8\end{array}$ & $\begin{array}{l}0.63 \\
5\end{array}$ & $\begin{array}{l}0.87 \\
1\end{array}$ & $\begin{array}{l}0.99 \\
1\end{array}$ & $\begin{array}{l}0.60 \\
0\end{array}$ & $\begin{array}{l}0.81 \\
4\end{array}$ & $\begin{array}{l}0.97 \\
1\end{array}$ & $\begin{array}{l}0.59 \\
4\end{array}$ & $\begin{array}{l}0.80 \\
2\end{array}$ & $\begin{array}{l}0.95 \\
1\end{array}$ \\
\hline & $\begin{array}{l}0 . \\
7\end{array}$ & $\begin{array}{l}0.94 \\
8\end{array}$ & $\begin{array}{l}0.98 \\
9 \\
\end{array}$ & $\begin{array}{l}0.99 \\
9 \\
\end{array}$ & $\begin{array}{l}0.94 \\
9 \\
\end{array}$ & $\begin{array}{l}0.98 \\
5 \\
\end{array}$ & $\begin{array}{l}0.99 \\
9 \\
\end{array}$ & $\begin{array}{l}0.95 \\
2 \\
\end{array}$ & $\begin{array}{l}0.98 \\
3 \\
\end{array}$ & $\begin{array}{l}0.99 \\
8\end{array}$ & $\begin{array}{l}0.96 \\
0 \\
\end{array}$ & $\begin{array}{l}0.98 \\
3 \\
\end{array}$ & 0.999 \\
\hline
\end{tabular}

\section{Conclusions:-}

In this paper we have discussed the test results of persistence change in presence of either a sudden or a timevarying variance break. Among our article we set the null hypothesis a unit root process with a variance shift occurs at some unknown point and the alternative hypothesizes are dented as $H_{01}$ and $H_{10}$. We use the statistics that derived by S. Leybourne, R. Taylor and T. H. Kim[2007]. The limiting distribution and divergence rate are given under both the null and alternative hypothesizes, separately. Moreover, simulation results show that there is serious size distortion and the power loss cannot be ignored either if the original statistics are applied without taking any measures.

\section{Appendix:-}

\section{Proof of theorem 3.1:-}

If assumption 1 and 2 holds and $\left\{y_{t}\right\}$ is a unit root process, easily we have:

$$
\bar{y}_{0}=[\tau T]^{-1}\left(y_{1}+y_{2}+\cdots+y_{[\tau T]}\right)=[\tau T]^{-1} \sum_{t=1}^{[\tau T]} \sum_{s=1}^{t} u_{s}
$$

According to the Lemma 1 of Giuseppe[2004], it is true that: 


$$
\begin{gathered}
T^{-1 / 2} \bar{y}_{0} \Rightarrow \tau^{-1} \int_{0}^{\tau} \bar{\sigma} \lambda_{\varepsilon} B_{\omega}(x) d x \\
\text { For } \sum_{i=1}^{[\tau T]} y_{t} \text {, we have: } \\
T^{-3 / 2} \sum_{t=1}^{[\tau T]} y_{t} \Rightarrow \int_{0}^{\tau} \bar{\sigma} \lambda_{\varepsilon} B_{\omega}(x) d x \\
\text { Similarly, we can get: } \\
T^{-2} \sum_{t=1}^{[\tau T]} y_{t}^{2} \Rightarrow \int_{0}^{\tau}\left(\bar{\sigma} \lambda_{\varepsilon} B_{\omega}(x)\right)^{2} d x \\
\text { For the numerator of } K^{f}(\tau), \text { we have: } \\
{[\tau T]^{-2} \sum_{t=1}^{[\tau T]} \hat{v}_{t, \tau}^{2}=[\tau T]^{-2} \sum_{t=1}^{[\tau T]}\left(y_{t}^{2}+\bar{y}_{0}^{2}-2 y_{t} \bar{y}_{0}\right)} \\
{[\tau T]^{-2} \sum_{t=1}^{[\tau T]} y_{t}^{2} \Rightarrow \tau^{-2} \int_{0}^{\tau}\left(\bar{\sigma} \lambda_{\varepsilon} B_{\omega}(x)\right)^{2} d x} \\
{[\tau T]^{-2} \sum_{t=1}^{[\tau T]} \bar{y}_{0}^{2} \Rightarrow \tau^{-3}\left(\int_{0}^{\tau} \bar{\sigma} \lambda_{\varepsilon} B_{\omega}(x) d x\right)^{2}} \\
{[\tau T]^{-2} \sum_{t=1}^{[\tau T]} 2 y_{t} \bar{y}_{0} \Rightarrow 2 \tau^{-3}\left(\int_{0}^{\tau} \bar{\sigma} \lambda_{\varepsilon} B_{\omega}(x) d x\right)^{2}}
\end{gathered}
$$

By the Lemma 4 of Giuseppe[2004], we have:

$$
\omega_{f}^{2}(\tau) \Rightarrow \bar{\sigma}^{2} \lambda_{\varepsilon}^{2}
$$

Thus, for $K^{f}(\tau)$, we have:

$$
K^{f}(\tau) \Rightarrow \tau^{-2} \int_{0}^{\tau} B_{\omega}(x)^{2} d x-\tau^{-3}\left(\int_{0}^{\tau} B_{\omega}(x) d x\right)^{2}
$$

For $K^{b}(\tau)$, a similar way can be applied and we only give the result here:

So,

$$
K^{b}(\tau) \Rightarrow(1-\tau)^{-2} \int_{\tau}^{1} B_{\omega}(x)^{2} d x-(1-\tau)^{-3}\left(\int_{\tau}^{1} B_{\omega}(x) d x\right)^{2}
$$

$$
R \Rightarrow \frac{\tau^{-2} \int_{0}^{\tau} B_{\omega}(x)^{2} d x-\tau^{-3}\left(\int_{0}^{\tau} B_{\omega}(x) d x\right)^{2}}{(1-\tau)^{-2} \int_{\tau}^{1} B_{\omega}(x)^{2} d x-(1-\tau)^{-3}\left(\int_{\tau}^{1} B_{\omega}(x) d x\right)^{2}}
$$

Where, $B_{\omega}(s)=\bar{\sigma}^{-1} \int_{0}^{s} \omega(r) d B(r), \bar{\sigma}^{2}=\int_{0}^{1} \omega(r)^{2} d r$.

\section{Proof of theorem 3.2:-}

We first consider 1 of the theorem for the results under $H_{01}$. When $\tau \leq \zeta$, all $\tilde{z}_{0, t}$ are $\mathrm{I}(0)$. Hence we have

Moreover

$$
\mathrm{T} \cdot[\tau T]^{-2} \sum_{t=1}^{[\tau T]} \tilde{z}_{0, t}^{2}=\tau^{-1} \cdot[\tau T]^{-1} \sum_{t=1}^{[\tau T]}\left(y_{t}-\bar{y}_{0}\right)^{2}
$$

$$
\sum_{t=1}^{[\tau T]}\left(y_{t}-\bar{y}_{0}\right)^{2}=\sum_{t=1}^{[\tau T]} y_{t}^{2}+[\tau T] \bar{y}_{0}^{2}-2 \bar{y}_{0} \sum_{t=1}^{[\tau T]} y_{t}=\sum_{t=1}^{[\tau T]} y_{t}^{2}-[\tau T] \bar{y}_{0}^{2}
$$

And

$$
[\tau T]^{-1} \sum_{t=1}^{[\tau T]} y_{t}^{2} \Rightarrow \bar{\sigma}^{2}
$$

According to the Lemma 1 of Giuseppe[2004], it is intuitively that

$$
T^{1 / 2} \bar{y}_{0}=T^{1 / 2}[\tau T]^{-1} \sum_{t=1}^{[\tau T]} y_{t} \Rightarrow \tau^{-1} \bar{\sigma} \lambda_{\varepsilon} B_{\omega}(\tau)
$$


As to consider the behavior of the variance estimator, basing on the proof of Theorem 2 of Leybourne[2007], we know it clearly that

$$
l \cdot \hat{\sigma}_{f}^{2}(\tau) \Rightarrow c
$$

And the probability limit $\mathrm{c}$ is finite and positive.

Combining the above results, we therefore have for $\tau \leq \zeta$

$$
T l^{-1} K^{f}(\tau) \Rightarrow \tau^{-1} c^{-1} \gamma_{0}^{*}
$$

Now consider the case where $\tau>\zeta$. We decompose the numerator of $K^{f}(\tau)$ into its constituent $\mathrm{I}(0)$ and I(1) part. Basing on the discussion above, we know that the $\mathrm{I}(0)$ part is asymptotically negligible.

$$
\begin{aligned}
{[\tau T]^{-2} \sum_{t=1}^{[\tau T]} \tilde{z}_{0, t}^{2}=} & \tau^{-2} T^{-2} \sum_{t=1}^{[\tau T]} y_{t}^{2}-\tau^{-3}\left(T^{-\frac{3}{2}} \sum_{t=1}^{[\tau T]} y_{t}\right)^{2} \\
& =\tau^{-2}\left(T^{-2} \sum_{t=1}^{[\zeta T]} y_{t}^{2}+T^{-2} \sum_{t=[\zeta T]+1}^{[\tau T]} y_{t}^{2}\right)-\tau^{-3}\left(T^{-\frac{3}{2}} \sum_{t=1}^{[\zeta T]} y_{t}+T^{-\frac{3}{2}} \sum_{t=[\zeta T]+1}^{[\tau T]} y_{t}\right)^{2} \\
= & \tau^{-2} T^{-2} \sum_{t=[\zeta T]+1}^{[\tau T]} y_{t}^{2}-\tau^{-3}\left(T^{-\frac{3}{2}} \sum_{t=[\zeta T]+1}^{[\tau T]} y_{t}\right)^{2}+o p(1) \\
\Rightarrow & \tau^{-2} \int_{\zeta}^{\tau} B_{\omega}(x)^{2} d x-\tau^{-3}\left(\int_{\zeta}^{\tau} B_{\omega}(x) d x\right)^{2}
\end{aligned}
$$

Next turn to the behavior of $\hat{\sigma}_{f}^{2}(\tau)$, observe that the $s$ th estimated autocovariance, $\hat{\gamma}_{s}$, can be expressed as a weighted average of $I(0)$ and $I(1)$ components as follows:

$$
\hat{\gamma}_{s}=\frac{\zeta}{\tau} \hat{\gamma}_{s, 0}+\left(1-\frac{\zeta}{\tau}\right) \hat{\gamma}_{s, 1}
$$

Where $\hat{\gamma}_{s, 0}$ is the sth estimated autocovariance when $y_{t}$ is $I(0)$; i.e.

$$
\hat{\gamma}_{s, 0}=[\zeta T]^{-1} \sum_{t=1}^{[\zeta T]} \Delta \hat{z}_{t, \tau} \Delta \hat{z}_{t-s, \tau}
$$

And $\hat{\gamma}_{s, 1}$ is the sth estimated autocovariance when $y_{t}$ is $I(1)$; i.e.

$$
\hat{\gamma}_{s, 1}=([T \tau]-[T \zeta])^{-1} \sum_{t=[T \zeta]+1}^{[T \tau]} \Delta \hat{z}_{t, \tau} \Delta \hat{z}_{t-s, \tau}
$$

Therefore, $\hat{\sigma}_{f}^{2}(\tau)$ can be decomposed commensurately as

$$
\hat{\sigma}_{f}^{2}(\tau)=\frac{\zeta}{\tau}\left(\hat{\gamma}_{0,0}+2 \sum_{s=1}^{m} w_{s, m} \hat{\gamma}_{s, 0}\right)+\left(1-\frac{\zeta}{\tau}\right)\left(\hat{\gamma}_{0,1}+2 \sum_{s=1}^{m} w_{s, m} \hat{\gamma}_{s, 1}\right)
$$

When $y_{t}$ is $I(0), \Delta y_{t}$ has an MA unit root so that

$$
\hat{\gamma}_{0,0}+2 \sum_{s=1}^{m} w_{s, m} \hat{\gamma}_{s, 0} \Rightarrow 0
$$

On the other hand, when $y_{t}$ is $I(1), \Delta y_{t}$ is stationary so that

$$
\hat{\gamma}_{0,1}+2 \sum_{s=1}^{m} w_{s, m} \hat{\gamma}_{s, 1} \Rightarrow \bar{\sigma}^{2}
$$

Hence we have

$$
\hat{\sigma}_{f}^{2}(\tau) \Rightarrow\left(1-\frac{\zeta}{\tau}\right) \bar{\sigma}^{2}
$$

Which means

$$
\hat{\sigma}_{f}^{2}(\tau)=O_{p}(1)
$$

Then for $\tau>\zeta, K^{f}(\tau)=O_{p}(1)$. To summarize, we obtain the limit function of $T l^{-1} K^{f}(\tau)$ is given by $\tau^{-1} c^{-1} \gamma_{0}^{*}$. $1\{\tau \leq \zeta\}+\infty \cdot 1\{\tau>\zeta\}$, which is therefore minimized at $\zeta$. Then we have 
Thus, $K^{f}(\tau)=O_{p}(l / T)$.

$$
\inf \left(T l^{-1} K^{f}(\tau)\right) \Rightarrow \tau^{-1} c^{-1} \gamma_{0}^{*}
$$

Next we consider the behavior of $K^{b}(\tau)$. For the case $\tau \leq \zeta$, we can write the denominator as

$$
\begin{gathered}
(T-[\tau T])^{-2} \sum_{t=[\tau T]+1}^{T} \tilde{z}_{1, t}^{2}=(T-[\tau T])^{-2} \sum_{t=[\tau T]+1}^{T} y_{t}^{2}-(T-[\tau T])^{-3}\left(\sum_{t=[\tau T]+1}^{T} y_{t}\right)^{2} \\
=(1-\tau)^{-2} T^{-2} \sum_{t=[\zeta T]+1}^{T} y_{t}^{2}-(1-\tau)^{-3}\left(T^{-\frac{3}{2}} \sum_{t=[\zeta T]+1}^{T} y_{t}\right)^{2}+o_{p}(1) \\
\Rightarrow(1-\tau)^{-2} \int_{\zeta}^{\tau} B_{\omega}(x)^{2} d x-(1-\tau)^{-3}\left(\int_{\zeta}^{\tau} B_{\omega}(x) d x\right)^{2}
\end{gathered}
$$

A similar discussion like above, it can be shown that the variance estimator, $\hat{\sigma}_{b}^{2}(\tau)$ is of $O_{p}(1)$. For the case when $\tau>\zeta$, all the $y_{t}$ are $I(1)$. Hence the limit of the denominator of $K^{b}(\tau)$ is given by

$$
\begin{gathered}
(T-[\tau T])^{-2} \sum_{t=[\tau T]+1}^{T} y_{t}^{2}-(T-[\tau T])^{-3}\left(\sum_{t=[\tau T]+1}^{T} y_{t}\right)^{2} \\
\Rightarrow(1-\tau)^{-2} \int_{\tau}^{1} B_{\omega}(x)^{2} d x-(1-\tau)^{-3}\left(\int_{\tau}^{1} B_{\omega}(x) d x\right)^{2}
\end{gathered}
$$

And $\hat{\sigma}_{b}^{2}(\tau)$ is again of $O_{p}(1)$. The above results imply that $\inf \left(K^{b}(\tau)\right)=O_{p}(1)$.

Combining the results all above, we get the first part of Theorem 2. As to the second part of Theorem 2, an analogous discussion can be applied and omitted.

\section{Reference:-}

1 Csörgö, M., Horváth, L. Invariance Principles for Change Point Problems[J]. Journal of Multivariate Analysis, 1988b, 27:151-168.

2 Csörgö, M., Horváth, L. Limit Theorems in Change-points Analysis[M]. John Wily and Sons Ltd Chichester, 1997.

3 Krishnaiah, P.R., Miao, B.Q. Review about Estimation of Change Point[J]. Handbook in Statistics, 1988, 7:375402.

4 Krishnaiah, P.R., Rao, C.R. Nonparametric Methods for Change Point Problems[J]. Handbook of Statistics, 1988, 243:403-425.

5 Kim, J. Y. Detection of change in persistence of a linear time series[J]. Journal of conometrics, 2000, 95:97116.

$6 \mathrm{Kim}, \mathrm{J}$. Y. Corrigendum to "detection of change in persistence of a linear time series"[J]. Journal of Econometrics, 2002, 109:389-392.

7 Yuandong, TU. Yanping, Yi. Forecasting Cointegrated Nonstationary Time Series with Time-Varying Variance[J]. Journal of Econometrics, 2016, 196(1):83-98.

8 Cavaliere, G. Taylor, A.M.R. Stationary Tests under Time- Varying Variances[J]. Econometric Theory, 2005, 21:1112-1129.

9 Cheng, X. Phillips, P.C.B. Cointegrating Rank Selection in Models with Time-Varying Variance. Journal of Econometrics, 2012, 169(2):155-165.

10 De Long, J. B., Summers, L. H. How does macroeconomic policy affect output[J]? Brookings Papers on Economic Activity, 1988, 2:433-494.

11 Hakkio, C. S., Rush, M. Is the budget deficit too large[J]? Economic Inquiry, 1991, 29:429-445.

12 Bai, J., Perron, P. Computation and Analysis of Multiple Structural Change Models[J]. Journal of Applied Econometrics, 2003, 18(1):1-22.

13 Bai, J., Robin, L.L., James, H.S. Testing For and Dating Common Breaks in Multiple Time Series[J]. Review of Economic Studies, 1998, 65:395-432.

14 Page, E.S. Continuous Inspection Scheme[J]. Biometrika, 1954,41(1/2):100-115.

15 Kokszka, P., Leipus, R. Change-point in the Mean of Dependent Observations[J]. Statistics and Probability Letters, 1998, 40(4):385-393. 
